\title{
Relationships between Nonlinear Normal Modes and Response to Random Inputs
}

\author{
Joseph D. Schoneman \\ Matthew S. Allen ${ }^{\dagger}$ \\ Engineering Physics Department, University of Wisconsin-Madison, Madison, Wisconsin, 53706, USA \\ Robert J. Kuether \\ Sandia National Laboratories ${ }^{1}$, Albuquerque, New Mexico, 87185, USA
}

\begin{abstract}
The ability to model nonlinear structures subject to random excitation is of key importance in designing hypersonic aircraft and other advanced aerospace vehicles. When a structure is linear, superposition can be used to construct its response to a known spectrum in terms of its linear modes. Superposition does not hold for a nonlinear system, but several works have shown that a system's dynamics can still be understood qualitatively in terms of its nonlinear normal modes (NNMs). This work investigates the connection between a structure's undamped nonlinear normal modes and the spectrum of its response to high amplitude random forcing. Two examples are investigated: a spring-mass system and a clamped-clamped beam modeled within a geometrically nonlinear finite element package. In both cases, an intimate connection is observed between the smeared peaks in the response spectrum and the frequency-energy dependence of the nonlinear normal modes. In order to understand the role of coupling between the underlying linear modes, reduced order models with and without modal coupling terms are used to separate the effect of each NNM's backbone from the nonlinear couplings that give rise to internal resonances. In the cases shown here, uncoupled, single-degree-of-freedom nonlinear models are found to predict major features in the response with reasonable accuracy; a highly inexpensive approximation such as this could be useful in design and optimization studies. More importantly, the results show that a reduced order model can be expected to give accurate results only if it is also capable of accurately predicting the frequency-energy dependence of the nonlinear modes that are in the frequency band of the excitation.
\end{abstract}

Keywords: Nonlinear Normal Modes, Geometric Nonlinearity, Random Response

\section{Introduction}

$T^{1}$ HE critical pressure loadings on the skin panels of concept hypersonic vehicles are random and broadband, resulting in response amplitudes that are so high that geometric nonlinearities cannot be neglected [1]. As a result, the dynamic response exhibits nonlinear phenomena such as increasing/decreasing resonance frequency with amplitude, energy transfer from low to high frequencies, etc... These same phenomena are also quite common in a variety of thin-walled structures, typically as the deflection magnitudes approach the shell thickness. In general, the response of such structures to random inputs cannot be computed analytically, but must be found via numerical integration of a finite element model. This is extremely computationally expensive even for relatively coarsely meshed structures, because long time records are required to obtain a reasonable estimate of the response statistics. Even more importantly, these simulations do not provide design engineers with much insight regarding how changes to specific deformation modes of the structure might affect its response. In the past few decades, reduced order modeling (ROM) techniques have been developed that can reduce large scale, nonlinear models to a low order

\footnotetext{
* Graduate Student, 534 Engineering Research Building, 1500 Engineering Drive, Madison, WI 53706-1609, schoneman@wisc.edu, AIAA Member.

${ }^{\dagger}$ Associate Professor, 535 Engineering Research Building, 1500 Engineering Drive, Madison, WI 53706-1609, msallen@engr.wisc.edu, AIAA Lifetime Member.

${ }^{\ddagger}$ Senior Member of Technical Staff, P.O. Box 5800, Albuquerque, NM, 87185, rjkueth@sandia.gov

${ }^{1}$ Sandia National Laboratories is a multi-program laboratory managed and operated by Sandia Corporation, a wholly owned subsidiary of Lockheed Martin Corporation, for the U.S. Department of Energy's National Nuclear Security Administration under contract DE-AC04-94AL85000.
} 
representation [2-4]. ROMs solve the problem of computational efficiency, but they model the nonlinear stiffness with a large number of polynomial coefficients and so they offer little intuition into the nonlinear behavior of a structure. The authors recently proposed that nonlinear normal modes (NNMs) could be used to gain insight into the dynamics of nonlinear structure; the NNMs have been shown [5] to provide an intuitive, qualitative description of a nonlinear system's free response and response to periodic forcing. This work builds on this foundation by exploring the connection between a structure's reduced order models, nonlinear normal modes, and its response to random inputs.

Nonlinear normal modes have received increased attention over the past several years. This work is primarily concerned with the definition provided by Vakakis, Kerschen and others $[5,6]$, who define an NNM as a notnecessarily synchronous periodic solution to the conservative nonlinear equations of motion. (This is an extension to the pioneering work of Rosenberg [7], who defined an NNM in essence as a vibration in unison of a conservative system.) One key feature of a NNM is the dependency of the system's natural frequency on response level. A common visual representation of this phenomenon is the frequency-energy plot, (FEP), on which a continuous backbone curve shows how the fundamental frequency (i.e. effective natural frequency) of the NNM changes with increasing response amplitude (or energy). For example, a geometrically nonlinear structure that stiffens with increased deflection will exhibit NNMs whose frequencies increase as the system energy increases.

The NNM definition used here captures internal resonances that result as underlying linear modes interact." These internal resonances are observed in the frequency-energy space as branches off of the main backbone curve, and they occur as two or more of the modes in the system interact, which may occur when the ratio between their NNM frequencies is rational (e.g. 3:1, 5:3, etc...). While many prior works have shown the effect that nonlinear stiffening has on the spectrum of the response (e.g. [1]), along with the relationship between the NNM backbones and harmonic forced response (e.g. [8]), it is unclear what effect these internal resonances have on the system response to random excitation, and whether they strongly influence the structure's behavior. Here, the influence of modal coupling and the resulting internal resonances is explored by comparing the response of various reduced order models which include or omit coupling terms between the modes. Additionally, random response autospectra of several structures are examined in conjunction with those structures' NNMs to study the connection between NNM backbone curves and the corresponding nonlinear frequency response.

The remainder of the paper is organized as follows: In section II, the theory behind reduced order models and nonlinear normal modes is presented. Section III details an investigation of a two degree of freedom system with a cubic nonlinearity, while section IV investigates the response of a beam with geometric nonlinearity. Finally, the conclusions are discussed in section V.

\section{Theoretical Development}

\section{A. Undamped Nonlinear Normal Modes}

The equations of motion for an undamped, homogeneous, $N$ degree-of-freedom geometrically nonlinear system can be written as

$$
\mathbf{M} \mathbf{K x}+\mathbf{f}_{N L}(\mathbf{x})=\mathbf{0}
$$

where $\mathbf{M}$ and $\mathbf{K}$ are the $N \times N$ linear mass and stiffness matrices, $\mathbf{f}_{N L}(\mathbf{x})$ is the $N \times 1$ nonlinear restoring force vector, and $\mathbf{X}$ is the displacement vector of the system. The structure's undamped NNMs are defined $[5,6]$ as the periodic solutions to this conservative equation of motion. The motion of each coordinate is periodic with a common fundamental frequency, but may also oscillate at higher harmonics as well. There is a clear analogy between this definition and the linear normal modes, which are periodic solutions assumed to have the form $\mathbf{x}=\boldsymbol{\varphi} \sin (\omega t)$ and found by solving an eigenvalue problem to obtain the natural frequency, $\omega$, and the mode shape, $\boldsymbol{\varphi}$ of each of the $N$ periodic responses that the linear system admits.

\footnotetext{
* In this paper we shall speak of linear and nonlinear modes and interactions between them because this type of description is helpful in understanding the physics of the system; we recognize that some may view this as a departure from the strict mathematical definitions of these terms.
} 
When the response amplitude of the nonlinear system is sufficiently low, the continuous branches of NNMs typically converge to the $N$ linear modes of the system*. (A system may have more than $N$ branches of NNMs. This will not be pursued here but it was discussed in $[9,10]$. The NNMs considered here are only the fundamental NNMs, or those NNMs that emanate from the linear modes at low energy.) The fundamental frequency and deformation shapes of these NNMs will vary with increasing energy. An example of this is shown in Fig. 1a, which gives the frequency-energy behavior of the two fundamental NNMs of a two-degree-of-freedom-system, and will be described in more detail in Section III. At the far left of the plot, each frequency-energy curve is flat; in this regime the NNM reduces to a linear mode of the structure and the NNM mode shape would be well approximated by the linear mode shapes. As energy (or response amplitude) increases, the frequency of the NNMs begins to increase, revealing that the nonlinearity has a hardening effect on these nonlinear modes. It is interesting to note the excursion in the frequency-energy curve for the first NNM near the 100 Joule energy level. At this point, the first NNM branch of the structure has a frequency that is precisely $1 / 3$ the frequency of the second; as a result, the two NNMs can be thought to interact. The solutions in this region show deformations where both the first and second linear mode shapes are present and the system may respond at the frequencies of both modes. This is termed an internal resonance [5].

\section{B. Connection Between Damped and Undamped Response}

The systems of interest in this work are damped and subjected to external forcing, so the conservative equation of motion (1) is modified by adding a damping matrix, which is assumed to be linear and light, and the external force vector $\mathbf{f}_{\text {ext }}(\mathbf{x}, t)$, which is a random function of time and position, is also included.

$$
\mathbf{M} \ddot{\mathbf{x}}+\mathbf{C} \dot{\mathbf{x}}+\mathbf{K x}+\mathbf{f}_{N L}(\mathbf{x})=\mathbf{f}_{e x t}(\mathbf{x}, t)
$$

This equation of motion is a nonlinear, second order Stochastic Differential Equation (SDE) [11]. It should be noted that this equation has received considerable attention recently as various researchers have sought to design nonlinear energy harvesting systems for random vibration environments (see, e.g. [12]). The equation above can be written in a form known as the Fokker-Planck equation [13], and its solutions can be found analytically for some low order systems, leading to further insights. Efficient solution strategies are also being developed for more general problems [13]. Cross and Worden recently studied a related approach for the SDOF version of this equation, revealing the level of approximation needed to capture different response phenomena, such as subharmonic peaks in the power spectra [14].

Although the NNMs are solutions to the homogeneous form in Eq. (1), they provide insight into the dynamics of the damped system when the damping is light and when it does not induce excessive coupling. This can be seen qualitatively by considering their relationship to the vector field described by Eqs. (1) and (2), as discussed in Appendix A. Furthermore, in steady state conditions the energy dissipated by the damping forces is balanced by the energy input by excitation, and Eq. (1) can be valuable in studying this condition, similar to the approach taken in $[15,16]$ to show that a single harmonic, single point force excites a damped system into a response that very closely resembles an NNM.

Returning to the application of interest, suppose that a randomly excited system is in quasi-equilibrium and the probability distribution of the energy (which is a random function of time) is known. We seek to investigate the following hypothesis.

Hypothesis: If the frequency energy plot (FEP) of a mode of interest and the probability density of the total energy in the structure are both known, then one can estimate the range of the response frequency in the vicinity of that mode simply by reading it off of the FEP at the appropriate energy level.

The authors do not claim that this hypothesis will hold rigorously, but it will be shown to provide a reasonable approximation that could be useful for design studies.

In order to study this hypothesis, a means must be devised for determining what fraction of a system's energy is stored within each nonlinear mode of the structure. In statistical mechanics and for linear systems, the Equipartition Theorem states that if a system is in thermal equilibrium then every mode will have the same mean square energy (over time) [17]. This concept has been successfully used to predict the mean square vibration of each mode of microcantilever beams due to temperature [18]. Unfortunately, nonlinear modes are not orthogonal and hence this theorem has not been extended to nonlinear systems. Even so, in the examples that follow we show that the

\footnotetext{
* Except when a system is essentially nonlinear, in which case some of the low energy modes may have zero fundamental frequencies.
} 
Equipartition Theorem is sometimes an excellent approximation for certain modes of a nonlinear structure. In other cases the spectrum is influenced by nonlinear couplings between the linear modes, which manifest themselves as internal resonances between the NNMs.

\section{Nonlinear Reduced Order Models}

In order to elucidate the connections between NNMs, and between approximations that include only the backbone or include both the backbone and nonlinear coupling between NNMs, it is helpful to construct reduced models that capture only one or just a few nonlinear modes. In a previous work it was shown that Reduced Order Models (ROMs) created using the Implicit Condensation and Expansion (ICE) method [2] can very accurately describe the backbone of flat, geometrically nonlinear structures; a single degree-of-freedom model was found to accurately capture the backbone of each NNM of interest in [19, 20]. In essence, these ROMs are simply linear modal models with added quadratic and cubic polynomial functions of the modal displacements. Because the method will be used extensively here it is explained briefly below.

First, the conservative, linear equations of motion are obtained by neglecting the nonlinear force vector and damping matrix in Eq. (2) and the classical eigenvalue problem is solved to obtain the modal natural frequencies, $\omega_{r}$, and linear mode shape matrix $\boldsymbol{\Phi}$. Then, the classical modal transformation,

$$
\mathbf{x}=\boldsymbol{\Phi q}
$$

is applied to Eq. (2) and the equation of motion for the $r^{\text {th }}$ mode then becomes

$$
\ddot{q}_{r}+2 \zeta_{r} \omega_{r} \dot{q}_{r}+\omega_{r}^{2} q_{r}+\theta_{r}\left(q_{1}, q_{2}, \ldots q_{m}\right)=\{\boldsymbol{\Phi}\}_{r}^{\mathrm{T}} \mathbf{f}_{e x t}(t)
$$

Prior works have shown that the geometric nonlinearity of thin-walled beams and plates $[1,4]$ can be effectively modeled using a polynomial model.

$$
\theta_{r}=\sum_{i=1}^{m} \sum_{j=1}^{m} B_{r}(i, j) q_{i} q_{j}+\sum_{i=1}^{m} \sum_{j=1}^{m} \sum_{k=1}^{m} A_{r}(i, j, k) q_{i} q_{j} q_{k}
$$

A distinct three-dimensional coefficient array $A_{r}(i, j, k)$ is associated with each mode in the ROM to account for cubic nonlinearities, and a two-dimensional array for the quadratic terms. These coefficients can be found by applying a set of static loads to the structure and then solving a least squares problem, as detailed in [2].

Once the ROM has been created, one can compute its nonlinear modes to gain insight into the dynamics of the structure. Various analytical/approximate methods exist, however in this work the continuation algorithm in [21] was used, which is implemented in the MATLAB ${ }^{\circledR}$ package "NNMcont", and can be accessed on Gaetan Kerschen's webpage*. The authors' prior works $[19,20]$ have shown that the NNMs computed from these ROMs can be made to be approximately equal to the corresponding NNMs of the full (unreduced) finite element model up to a certain energy level if the appropriate basis vectors and load scaling factors are used to construct the ROM. See [19, 20] for further details. When this is the case, the response of that ROM can be studied to understand how the structure would respond if its dynamics were reduced to only the degrees of freedom necessary to reproduce that subset of the NNMs. This approach is used in the following examples to elucidate the connection between the NNMs and various features that are observed in the random response.

\section{Motivation - Academic Test Case}

The connection between a structure's nonlinear modes and its random response is first explored for a simple 2DOF system that is shown in Fig. $1 \mathrm{~b}$ and whose equations of motion are given below.

$$
\left[\begin{array}{cc}
m_{1} & 0 \\
0 & m_{2}
\end{array}\right]\left\{\begin{array}{l}
\ddot{x}_{1} \\
\ddot{x}_{2}
\end{array}\right\}+\beta\left[\begin{array}{cc}
k_{1} & -k_{1} \\
-k_{1} & k_{1}+k_{2}
\end{array}\right]\left\{\begin{array}{l}
\dot{x}_{1} \\
\dot{x}_{2}
\end{array}\right\}+\left[\begin{array}{cc}
k_{1} & -k_{1} \\
-k_{1} & k_{1}+k_{2}
\end{array}\right]\left\{\begin{array}{l}
x_{1} \\
x_{2}
\end{array}\right\}+\left\{\begin{array}{l}
k_{n l, 1}\left(x_{1}-x_{2}\right)^{3} \\
k_{n l, 2}\left(x_{2}-x_{1}\right)^{3}
\end{array}\right\}=\left[\begin{array}{c}
f_{e x t}(t) \\
0
\end{array}\right]
$$

The damping is stiffness-proportional with $\beta=0.02$ and for all cases studied here the parameters of the system are: $m_{1}=m_{2}=1, k_{1}=1, k_{2}=0.2$, and the cubic nonlinear spring coefficients are $k_{\mathrm{nl}, 1}=0.5$ and $k_{\mathrm{nl}, 2}=1 \mathrm{e}-5$. Figure $1 \mathrm{a}$ shows the frequency-energy dependence of the system's two primary nonlinear normal modes. This system is linearizable and the (constant with energy) natural frequencies of the corresponding linear modes are shown with dotted lines. The nonlinear mode deformation shapes are not shown here, but it was observed that, the linear mode

\footnotetext{
${ }^{*}$ http://www.ltas-vis.ulg.ac.be/cmsms/index.php?page=kerschen (Accessed June 2013)
} 
shapes of the system show similar motion of each of the masses (in phase and out of phase for the $1^{\text {st }}$ and $2^{\text {nd }}$ modes respectively), while the NNM motion localizes to one or the other of the masses as energy increases.
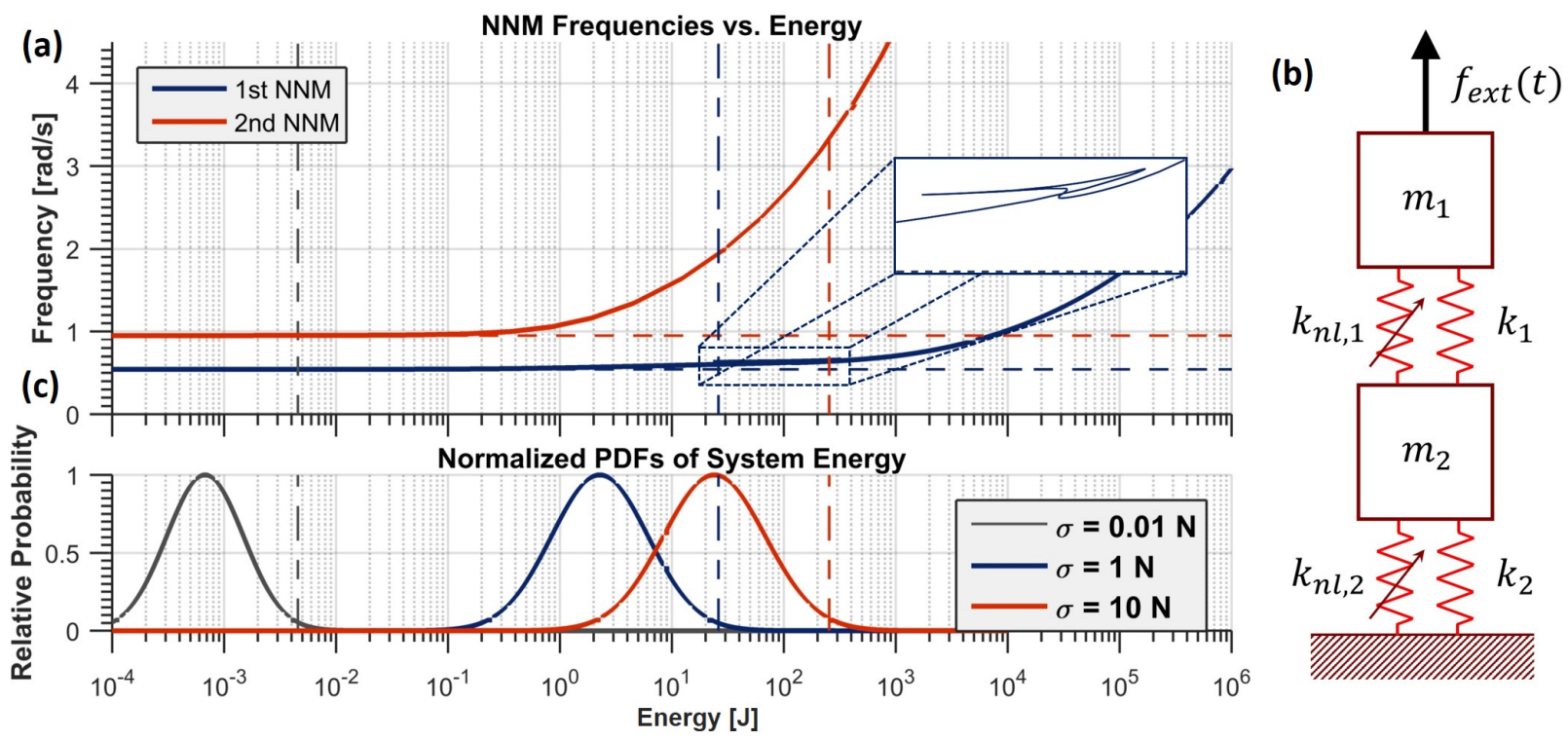

Figure 1. (a) Frequency-energy plot of the 2DOF system pictured in the schematic (b). (c) Estimated lognormal probability distributions of the instantaneous energy level in each system. Vertical dashed lines in (a) and (c) indicate the $95^{\text {th }}$ percentile of each energy PDF; horizontal dashes in (a) denote linear natural frequencies.

The hypothesis discussed in Section II.B was evaluated by applying a number of random broadband inputs to this system. The first mass was forced by a broadband, Gaussian random input force, $f_{\text {ext }}(t)$, which is zero-mean with standard deviation $\sigma$. The response of the system to this input was computed using an adaptive $4^{\text {th }} / 5^{\text {th }}$ order Runge-Kutta time integration routine (i.e. MATLAB's 'ode45' function). The input was generated using three different forcing levels, and, to facilitate the calculations, filtered with an $8^{\text {th }}$ order, low-pass Butterworth filter with a cutoff frequency of $5 \mathrm{rad} / \mathrm{s}$. The response was computed over a 10,000 second time interval in order to guarantee statistical convergence. Post-simulation, the instantaneous energy level of the system was computed at each instant in time and then that vector of energies was interrogated and found to resemble a log-normal distribution. MATLAB's statistical toolbox was then used to estimate the probability density function (PDF) of the energy for each case; the resulting PDFs (normalized to a maximum value of one, for comparison) of the system energy are shown in Fig. 1c, with the dashed vertical lines corresponding to the 95th percentile of each distribution. The three cases can be seen to correspond to low energy where the system is essentially linear (gray), medium energy where the nonlinearity begins to have a noticeable effect (blue), and very high nonlinearity (red). In the final case the second natural frequency has shifted by a factor of four or more and an internal resonance in the first NNM is encompassed, near the $100 \mathrm{~J}$ energy level. The frequencies at which each 95th percentile line intercepts the first and second NNMs, along with numerical values for the input and output of each case, are given in Table 1.

Table 1: Details of the various simulated load cases for the 2DOF system

\begin{tabular}{|c|c|c|c|}
\hline $\begin{array}{c}\text { Force Std. Dev. } \boldsymbol{\sigma} \\
{[\mathbf{N}]}\end{array}$ & $\begin{array}{c}\text { 95th Percentile Response } \\
\text { Energy [J] }\end{array}$ & NNM 1 Intercept [rad/s] & NNM 2 Intercept [rad/s] \\
\hline 0.01 & $4.56 \cdot 10^{-3}$ & 0.54 & 0.95 \\
\hline 1 & 26.06 & 0.61 & 1.90 \\
\hline 10 & 254.6 & 0.64 & 3.30 \\
\hline
\end{tabular}


The power spectral densities of the responses were then computed and are shown in Fig. 2. It is interesting to note how well the frequency content in each spectrum corresponds with that predicted using the proposed hypothesis. For example, for the moderate excitation (blue lines in Fig. 1c/Fig. 2), 95\% of the system energy lies below $26.06 \mathrm{~J}$; at this point the frequency of NNM-1 varies by only about $5 \%$ and the slope of the NNM backbone has barely begun to increase. Corresponding with these observations, the first peak in the autospectrum has not shifted or spread significantly, showing about the same shape that it did for the low-amplitude input. In contrast, the second NNM features a significant frequency shift at this energy level, and the backbone curve has a much larger slope. Examining the second peak in the spectrum in Fig. 2, it is clear that the peak has shifted and spread considerably compared to the linear response. Similar comments could be made about the comparison when the system was excited at higher and lower energies. Furthermore, as the forcing amplitude increases the response near each peak also localizes to DOF 2 and 1 respectively, similar to the manner in which the NNMs localize to these DOF at high energy. It is somewhat surprising that the random response of a system such as this, which can potentially exhibit complicated phenomena such as internal resonance, subharmonic resonance, etc... would be so readily described by the frequency-energy dependence of its NNMs as was shown here. Furthermore, the frequency content near each nonlinear mode of the structure seems to be consistent with what one would expect based on the Equipartition Theorem.

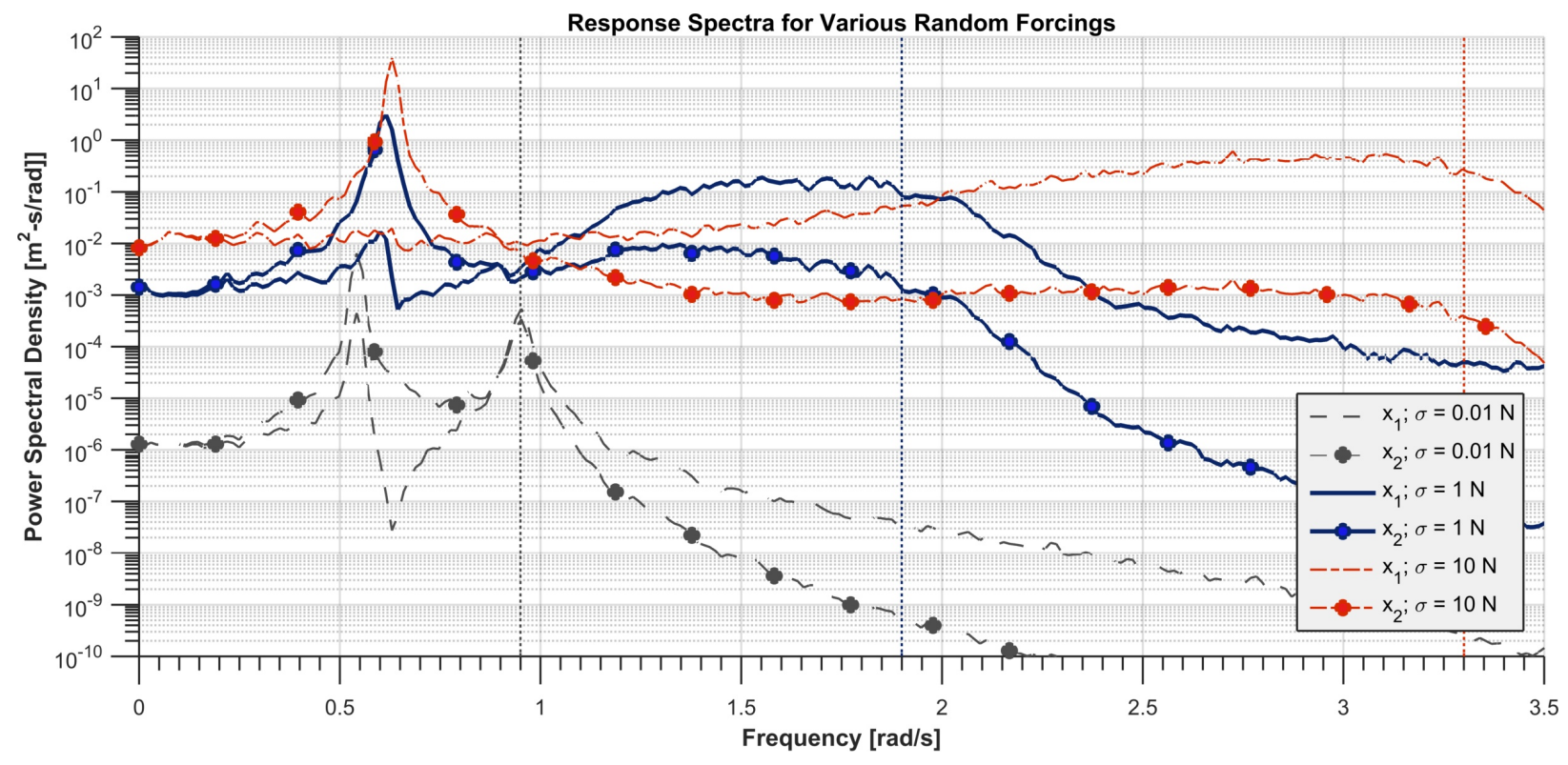

Figure 2. Power Spectrum of the response of the 2DOF system at various forcing levels. Vertical dotted lines correspond to the 95th percentile energy intercept of NNM 2 for each load case - see Figure 1/Table 1. NNM 1 intercepts are omitted for clarity.

\section{Case Study - Clamped-Clamped Beam}

\section{Base Excitation Case}

To explore these concepts further, the response of a clamped-clamped beam was examined. The structure was modeled with $40 \mathrm{~B} 31$ beam elements in the Abaqus ${ }^{\circledR}$ finite element package and has the physical properties listed in Table 1. The beam was subjected to a random, zero-mean Gaussian base excitation with a standard deviation of $8 g$ and a bandwidth of 0 to $2000 \mathrm{~Hz}$, making this case identical to one that was studied by Gordon and Hollkamp in [1]. 
Table 2: Physical properties of the clamped-clamped beam

\begin{tabular}{|c|c|c|c|c|c|}
\hline Length & Width & Thickness & $\begin{array}{c}\text { Young's } \\
\text { Modulus }\end{array}$ & Shear Modulus & \multicolumn{2}{|c|}{ Mass Density } \\
\hline $9 \mathrm{in}$ & $0.5 \mathrm{in}$ & $0.031 \mathrm{in}$ & $2.97 \cdot 10^{7} \mathrm{psi}$ & $1.16 \cdot 10^{7} \mathrm{psi}$ & $7.36 \cdot 10^{-4} \frac{\mathrm{lb} \cdot \mathrm{s}^{2}}{\mathrm{in}^{4}}$ \\
\hline
\end{tabular}

Several reduced order models were created which capture a subset of the system's dynamics. Due to the form of the excitation, only symmetric (odd) modes need be considered for inclusion in the ROM. The ROMs were constructed using the ICE method with only cubic nonlinear terms, and using a set of load cases that would displace the linearized beam statically by one beam thickness. This load level was used in $[20,22]$ and found to give excellent predictions for the first several NNMs of this structure. The first three odd NNMs of the system are plotted in Fig. 3 with comparisons between single-mode (1) ROMs, a four-mode $(1,3,5,7)$ ROM, and those computed from the full order model with the Applied Modal Force (AMF) algorithm in [19]. The AMF algorithm iterates on the full finite element model in the native code by adjusting the initial conditions until a periodic response is identified, and hence the solutions that it obtains are true NNMs (to a certain numerical tolerance). For reference, the modal deformation shapes for the first NNM are shown in Fig. 4. These reveal that the deformation shape of NNM 1 remains largely unchanged along the backbone (although the level of axial strain changes dramatically revealing that high-order axial modes are statically coupled to this mode [1]). The shape also changes dramatically near an internal resonance, where it interacts with other modes.

The results show that single-mode ROMs reproduce the NNMs quite accurately except near the internal resonances. Also, the single-mode curve does overestimate the frequency slightly near the upper end of the energy range that is shown for each mode. Since each of the single-mode ROMs captures the response of a single nonlinear mode reasonably well, they can be used to probe the degree to which the response of the system can be predicted (or, cannot be predicted) using a linear superposition of the nonlinear normal modes. This will be explored in the results that follow.

A four-mode $(1,3,5,7)$ ROM is also shown, which agrees very well with the truth NNM over the frequency and energy ranges shown in Fig. 3. This ROM includes the coupling between the modes, so it also reproduces the internal resonances along the $1^{\text {st }} \mathrm{NNM}$ backbone curve, which occur at 88 and $125 \mathrm{~Hz}$. (The AMF algorithm was intentionally set to skip over the internal resonances, to avoid the computational expense required to compute them, so their accuracy is not verified here, but in [19] a solution is shown which includes the internal resonances and they do agree well with those predicted by the ROM in Fig. 3.) Hence, the four mode ROM can be used to obtain an accurate estimate of the true response of the beam, including the coupling between these four modes.

Because this model contains only 40 elements, it was also possible to integrate the full order finite element model of this beam within Abaqus ${ }^{\circledR}$. While modal damping is convenient to use within the ROM formulation, Abaqus does not allow one to define modal damping ratios in a nonlinear transient simulation. Hence, mass and stiffness proportional damping with $\mathbf{C}=\alpha \mathbf{M}+\beta \mathbf{K}$, was used instead in the full-order model. In [1], it was noted that the damping ratios of the first two symmetric modes were experimentally observed to be $\zeta_{1}=0.3 \%$ and $\zeta_{3}=$ $0.5 \%$. To maintain comparable values over the $2000 \mathrm{~Hz}$ frequency range of interest, these damping ratios were imposed at frequencies of $78 \mathrm{~Hz}$ and $2000 \mathrm{~Hz}$. The proportional damping coefficients of $\alpha=4.77$ and $\beta=7.77 \cdot 10^{-7}$ were selected to fulfill this requirement. 

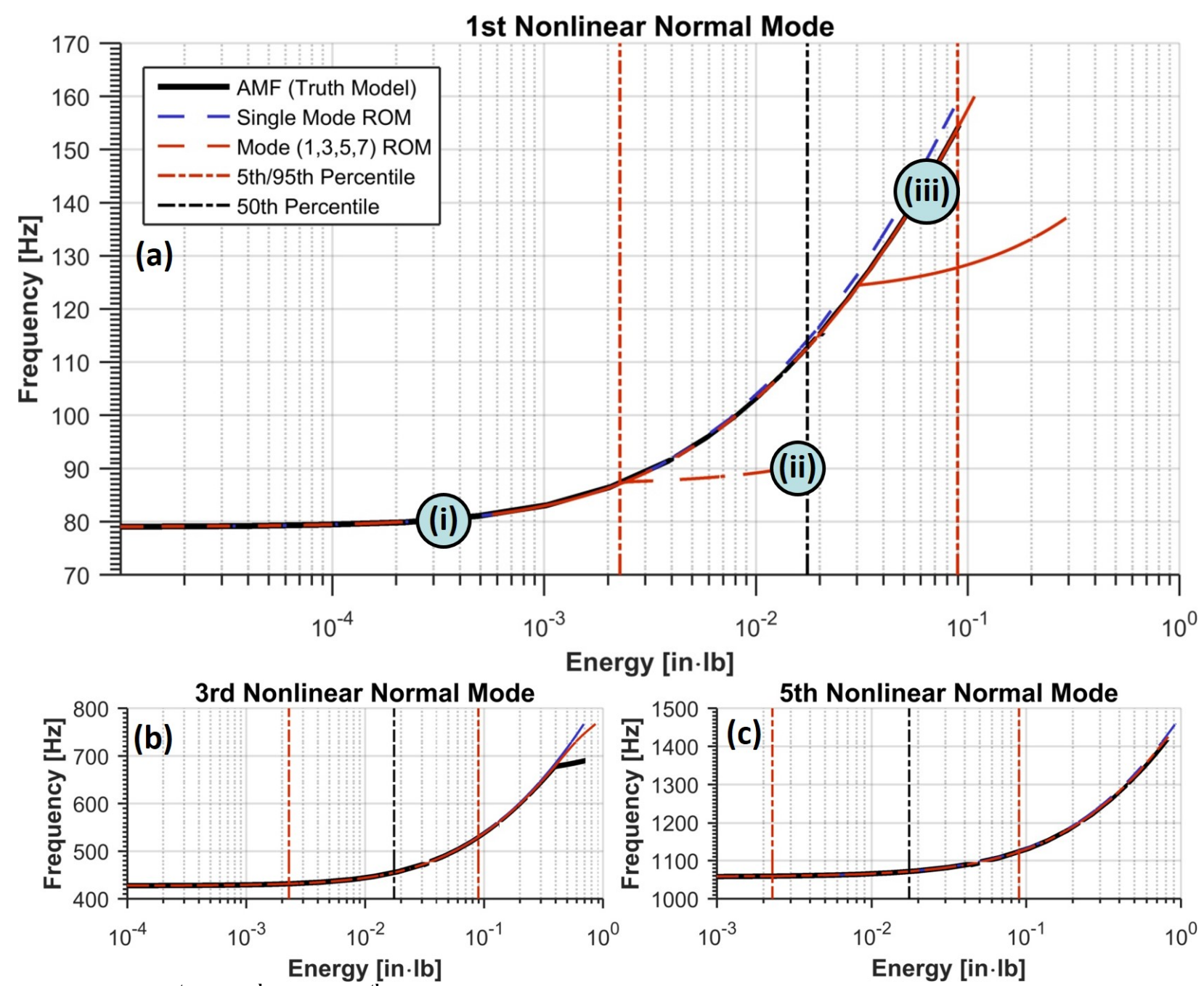

Figure 3. (a) $1^{\text {st }}$, (b) $3^{\text {rd }}$, and (c) $5^{\text {th }}$ NNMs of the clamped-clamped beam. Vertical line indicates the energy percentile levels in the random response - see Figure 5. For reference, a displacement amplitude of one beam thickness corresponds to $E=0.006,0.3$ and 1.2 in-lb respectively for modes 1,3 and 5 .

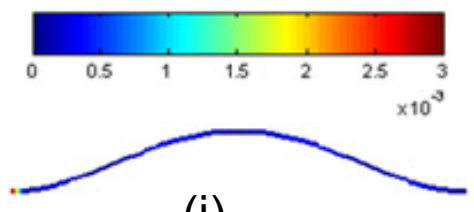

(i)

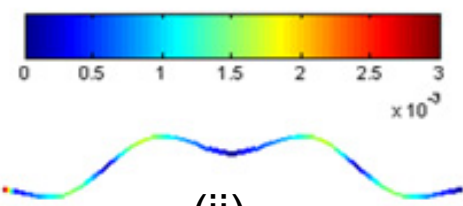

(ii)

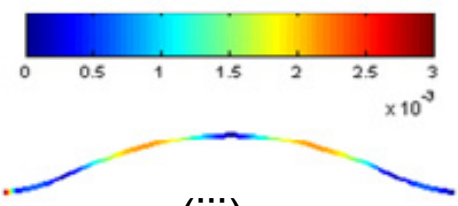

(iii)

Figure 4. Deformation shapes at three points along the $1^{\text {st }} \mathrm{NNM}$, as marked in Fig. 3. The colorbar shows the ratio of the bending displacement to axial displacement, revealing that axial stretch in the beam becomes more important as energy increases.

MATLAB was used to define the amplitude of the random base acceleration at each node in the finite element model at a fixed time-step. Based on the results of a convergence study, a sample rate of $50 \mathrm{kHz}$ was found to be sufficient to accurately integrate the finite element model. To obtain statistically significant results, a relatively long time history was required; a 25 second simulation was used which yielded 1,250,000 time points. The full finite element model took roughly 12 hours to integrate on a $3.4 \mathrm{GHz}$ Intel® Core $i 7$ with $12 \mathrm{~GB}$ of RAM. To examine the response of the reduced order models, the Newmark integration method was implemented within MATLAB and used with the same base acceleration profile that was supplied to Abaqus (this integration took only 1-2 minutes). Then, the most accurate of these results, the 4-mode ROM containing modes 1, 3, 5, and 7, was used to compute the 
beam's energy distribution over the integration period. This was used to create a histogram of the instantaneous energy level and used to create a probability mass function (PMF) giving the discrete probabilities of each energy value; this is shown with gray dots in Fig. 5. As was the case with the two-degree-of-freedom system above, the distribution resembles a bell curve on a logarithmic scale, so it was approximated with a log-normal distribution. The resulting curve, along with its 5th, 50th and 95th percentile energy levels, is also plotted in Fig. 5. Frequency intercept locations for each NNM and percentile level are listed in Table 3.

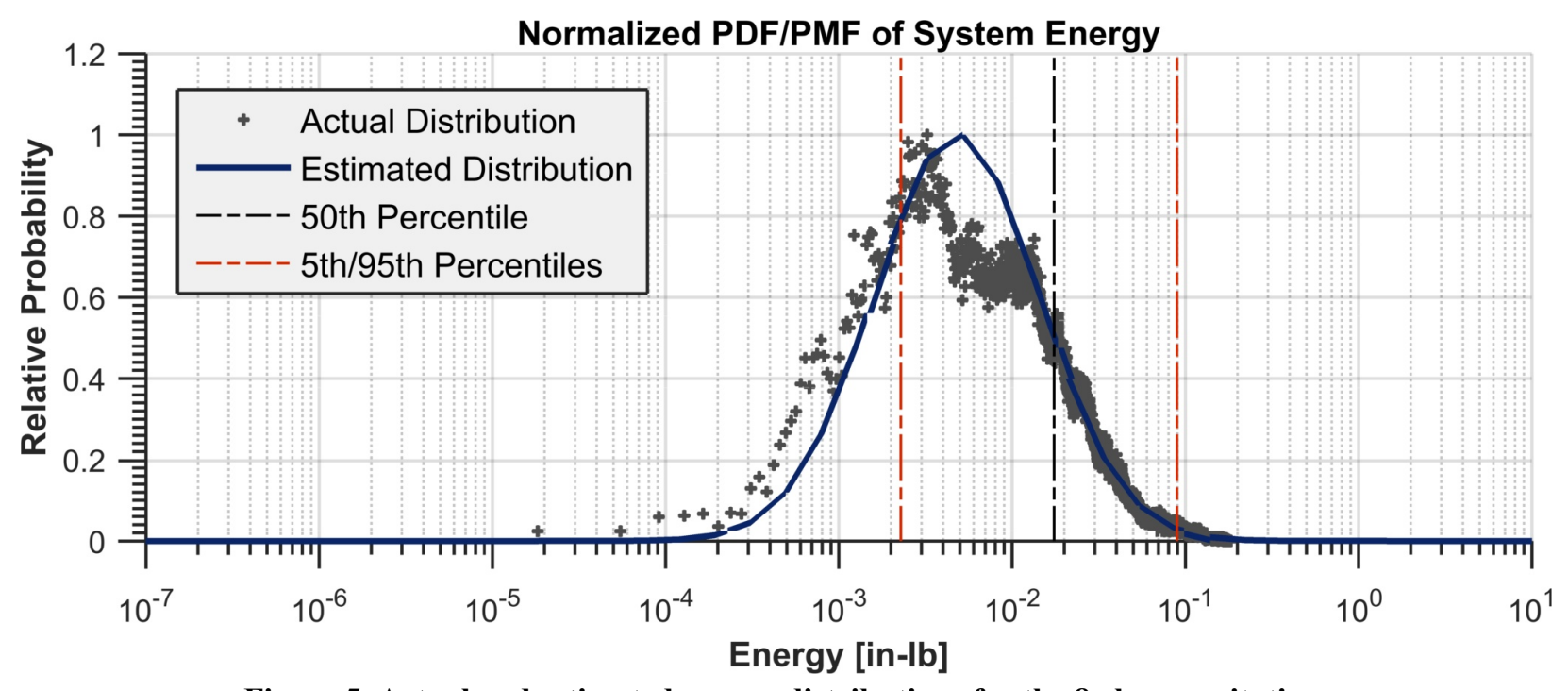

Figure 5. Actual and estimated energy distributions for the 8g base excitation.

Table 3: Energy percentile levels and frequency intercepts for $8 \mathrm{~g}$ base excitation.

\begin{tabular}{|c|c|c|c|c|}
\hline Percentile & Energy Level [in-lb] & $\begin{array}{c}\text { NNM 1 Intercept } \\
{[\mathbf{H z}]}\end{array}$ & $\begin{array}{c}\text { NNM 2 Intercept } \\
{[\mathbf{H z}]}\end{array}$ & NNM 3 Intercept [Hz] \\
\hline 5 & 0.00228 & 87 & 431 & 1050 \\
\hline 50 & 0.0175 & 113 & 455 & 1070 \\
\hline 95 & 0.0896 & 154 & 530 & 1120 \\
\hline
\end{tabular}

The power spectral density (PSD) of the response of the various models is displayed in Figure 6. The spectrum of the truth model, obtained by integrating the full finite element model, is shown in each subplot with a solid black line. Figure 6a displays the response of the $(1,3,5,7)$ ROM compared to the response of the full-order model, confirming that this ROM accurately predicts the response to a random base excitation. The frequency-energy plots in Fig. 3 and the energy distribution in Fig. 5 were used to find the frequency corresponding to each energy percentile. The vertical lines in Fig. 6a show the frequencies of the $5^{\text {th }}$ and $95^{\text {th }}$ percentile of the response energy distributions. Interestingly, the center of each peak lies near the $50^{\text {th }}$ percentile marker, while the distribution of each peak is found to be bracketed by the $5^{\text {th }}$ and $95^{\text {th }}$ percentile frequencies. Once again this supports the hypothesis that the frequency observed near an NNM can be estimated using the Equipartition Theorem, even for a highly nonlinear structure such as this.

Next we compare the results of the full order model with the response of a reduced model that captures only one or two of the nonlinear modes in the bandwidth of interest. Figure $6 \mathrm{~b}$ compares the response of the single-mode ROMs generated with either mode 1,3 , or 5 . The mode 1 ROM was found to predict the first peak in the spectrum quite accurately, while the ROMs that included only mode 3 and mode 5 are noticeably too sharp (i.e. their nonlinearities are not active and so their peaks do not spread). This is an interesting finding; it reveals that when the random load is applied to a ROM that captures only mode 3 (or mode 5), the one-mode system does not absorb enough energy to transition into the nonlinear regime. More specifically, the energy absorbed must be small enough that the NNM frequency shown in Fig. 3(b) (or 3(c) for mode 5) does not increase significantly. In contrast, if we 
consider the total energy absorbed by the full-order model, whose mean and quantiles are shown in Fig. 3(b) (or 3(c)), then we expect each mode's peak in the PSD to smear considerably, as was illustrated in Fig. 6(a). Hence, the Equipartition Theorem proved to be more useful in predicting the smearing in the PSD than the uncoupled one-mode ROMs.

Further insight can be obtained by considering the response of various two-mode ROMs. Figure 6(c) shows the response predicted from two-mode ROMs created using modes $(1,3)$. This ROM captures the backbones of NNMs 1 and 3 and the associated internal resonance near $88 \mathrm{~Hz}$ in Fig. 3(a). (The frequency energy plot for this $(1,3)$ mode ROM, which is not shown here, was nearly identical to that of the $(1,3,5,7)$ ROM shown in Fig. 3(a) except that it did not include the second internal resonance near $125 \mathrm{~Hz}$.). Figure 6(c) shows that this two-mode model predicts the random response in the frequency range of NNMs 1 and 3 very well. However, it is interesting to note that the internal resonance near $88 \mathrm{~Hz}$ does not seem to produce any discernible feature in the spectrum in Fig. 6(a) or 6(c). The simulations were repeated at a variety of force levels and none showed a discernible feature in the spectrum near $88 \mathrm{~Hz}$. Hence, while it is clear that the presence of an internal resonance does reveal which modes in a ROM are coupled, it is not clear whether it produces any features in the spectrum. In contrast, there seems to be a clear connection between the backbone (sometimes called the primary NNM branch) and the features in the spectrum of the response.

These observations may lead one to conclude that modal coupling in the ROM has allowed energy transfer between modes 1 and 3 and that this produces the spreading that is observed in the spectrum near mode 3 . This may be true, yet the authors discovered that the observed result could also be explained using a set of linear singledegree-of-freedom models. Specifically, the structure was deformed statically into its first mode of vibration with the instantaneous amplitude of linear mode 1 , and then the $3^{\text {rd }}$ and $5^{\text {th }}$ linear natural frequencies were computed about that deformed state. The deformation in mode 1 induces tension in the beam which increases (stiffens) the $3^{\text {rd }}$ and $5^{\text {th }}$ natural frequencies somewhat. This process was then used to estimate the distribution of the mode 3 and 5 frequencies given the known distribution of pre-stress in mode 1 and it was found to predict the width of the PSD near modes 3 and 5 very accurately. That calculation is discussed in Appendix B and the result is presented for the case presented in the following section. This suggests that the spreading of the spectrum near modes 3 and 5 may simply come from the tension in the beam induced by large deformation in mode 1, rather than due to an actual transfer of energy transfer to those modes. 

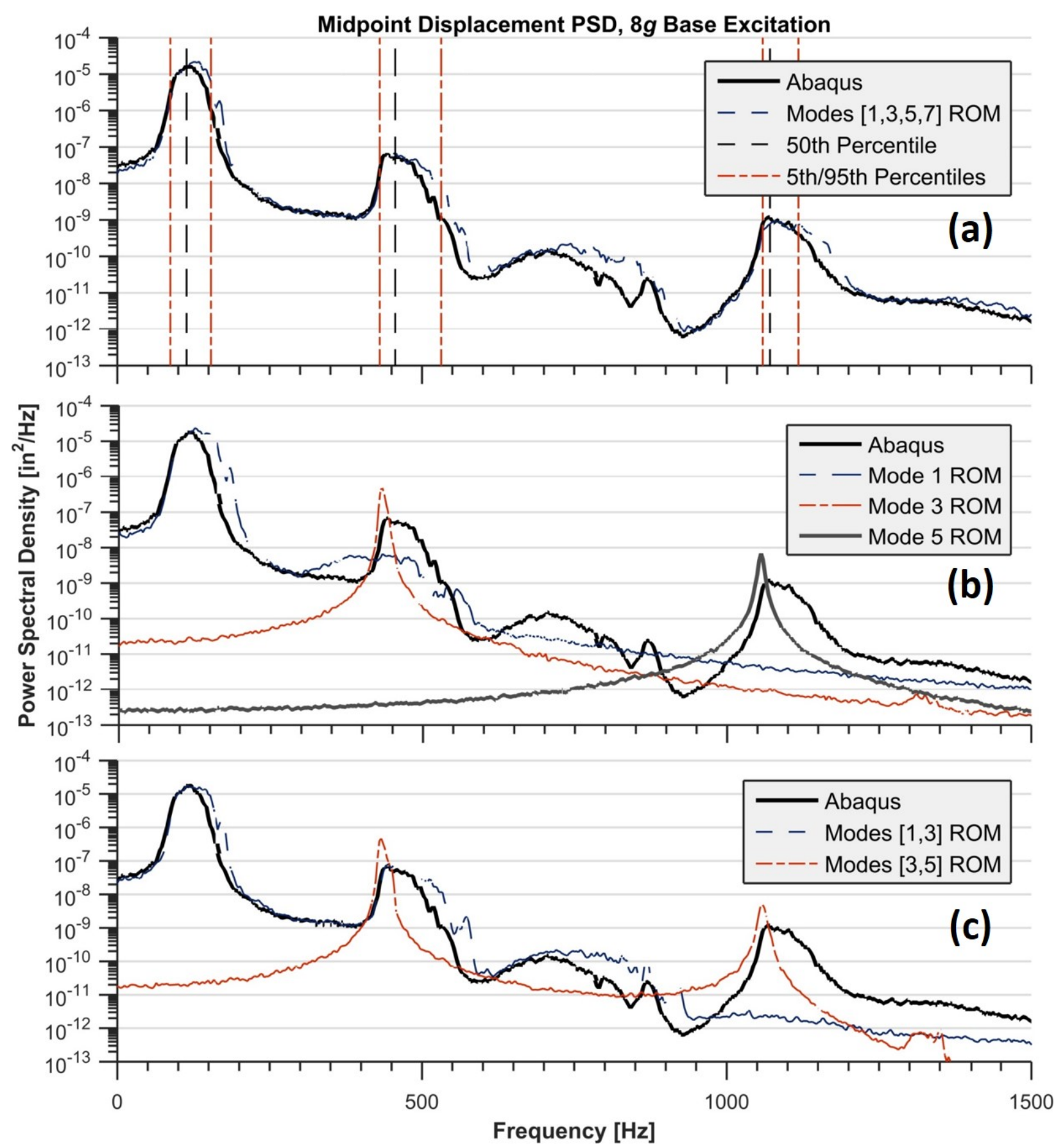

Figure 6. Power spectral density estimates for various ROMs compared to the Abaqus truth model. (a) Fourmode ROM, (b), single-mode ROMs, (c), two-mode ROMs.

These results suggest that, while a specific mode must be included in the ROM in order to obtain the corresponding response peak, the response as a whole may be inaccurate if a particularly dominant mode is left out. In this case, mode 1 is dominant, due to the form of the excitation, so its effect on the structure must be considered to correctly predict the response even at frequencies that are significantly higher.

To understand how these differences might affect a life prediction, the root mean square (RMS) strain at the center of the beam was computed using a few of the ROMs and the result is shown in Fig. 7. To separate the effect of modes 1 and 3, the RMS was computed by integrating the region under the peaks in the PSD near modes 1 and 3, e.g. from $30-180 \mathrm{~Hz}$ and $350-600 \mathrm{~Hz}$ respectively (i.e. in Fig. 6). The results show that the strain near mode 1 
increases at a much lower rate than what a linear model would predict (black dashed line). In contrast, the strain increases approximately linearly near mode 3 . The mode 1 ROM provides an excellent estimate of the strain in the beam up to about $6 \mathrm{~g}$, and even at the highest levels considered it is within $17 \%$ of that predicted by the mode $(1,3,5,7)$ ROM (which is very similar to the full finite element model). The mode 3 ROM is even more accurate over the whole range of base accelerations, which is surprising considering the fact that it produces a spectrum that is noticeably in error (see Fig. 6b). On the other hand, since it appears that mode 3 is behaving linearly about the state induced as mode 1 stretches the beam, perhaps it is reasonable that a linear model has the correct mean square amplitude. This further supports the notion that there is not significant energy transfer between modes, but simply a nonlinear response of mode 1 that affects the dynamics of the other modes.

The results show that, for this system, once one has a ROM that captures the NNM backbone accurately, the strain that the system will experience in a random environment can be estimated fairly well over a range of forcing amplitudes by simply integrating a single second-order differential equation.

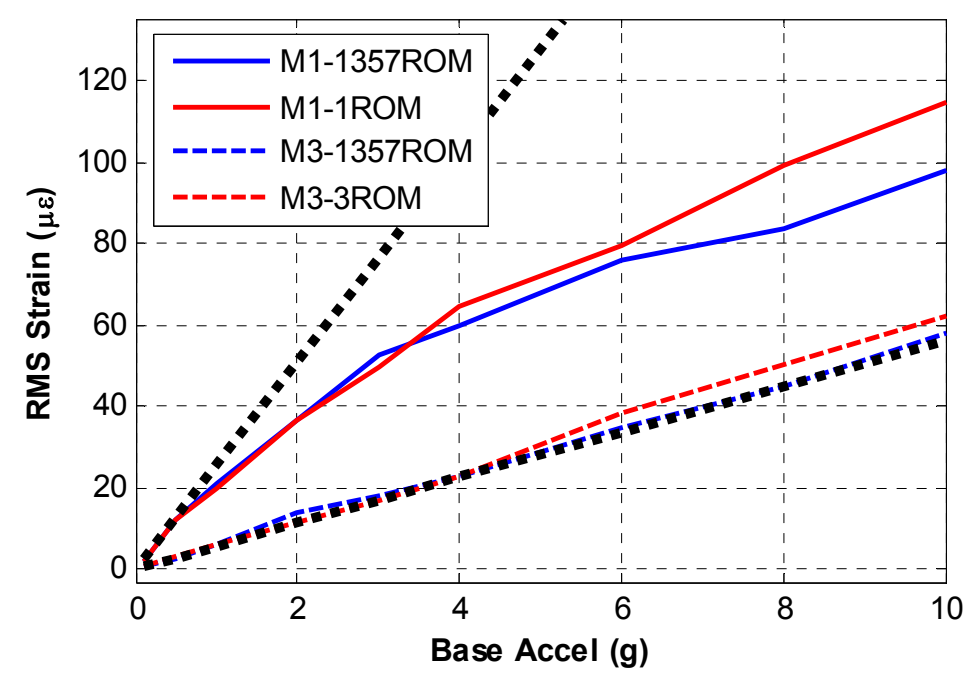

Figure 7. Root mean square strain at the surface of the center of the beam versus the RMS base acceleration. Dashed black lines depict predictions obtained using linear models for modes 1 and 3 respectively.

\section{E. Random Pressure Field Excitation}

In the applications of interest, structures are excited by a random pressure field, so one further load case was considered to see the effect of a more general loading. Here, a forcing was used that is a random function of both position and time. A correlation matrix was defined such that,

$$
E\left[\mathbf{y} \mathbf{y}^{\mathrm{T}}\right]=\mathbf{C} \text { where } C_{i j}=\exp \left[-\alpha\left|x_{i}-x_{j}\right|\right]
$$

with $E[]$ the expected value operator, $\alpha$ a positive constant and $\left|x_{i}-x_{j}\right|$ the distance between the $i^{\text {th }}$ and $j^{\text {th }}$ points. In the continuous sense, $\mathbf{y}$ is a vector of correlated random forcing functions, with each random element corresponding to a nodal force. To implement this field it is convenient to create a matrix $\mathbf{Y}$ which has a column for each time instant. If the model contains $q$ nodes and is simulated at $r$ time steps, $\mathbf{Y}$ has dimension $q \times r$. To find this correlated random field, one first obtains the lower triangular Cholesky decomposition of $\mathbf{C}$, denoted as $\mathbf{b}$, so that $\mathbf{C}=\mathbf{b}^{\mathrm{T}} \mathbf{b}$. Then, given a matrix $\mathbf{W}$ of $q$ independent variables at $r$ time steps, the random time history can then be found using Equation (8).

$$
\mathbf{Y}=\mathbf{b W}
$$

In the case that follows, the initial random matrix $\mathbf{W}$ consisted of independent, Gaussian random variables with zero mean and standard deviations of $0.08 \mathrm{lb}$. The 25 second time histories were sampled at $50 \mathrm{kHz}$ and filtered through an 8th order low-pass Butterworth filter with a critical frequency of $2000 \mathrm{~Hz}$. A correlation constant of $\alpha=0.5$ was used to obtain the correlation matrix. In this manner, the set of initially independent vectors were "smoothed" across the beam, to provide a forcing reminiscent of physically realizable loadings. Due to the 
asymmetric nature of this loading scenario, both even and odd linear modes were excited and will be used in the construction of the ROMs for this case.

As above, the energy distributions were first determined and are shown in Fig. 8. For this case, the distribution was computed using a ROM that contained modes one through four. The second and fourth NNMs had converged to the AMF solution, are quite similar to their first, third and fifth NNM counterparts, and are thus not displayed.

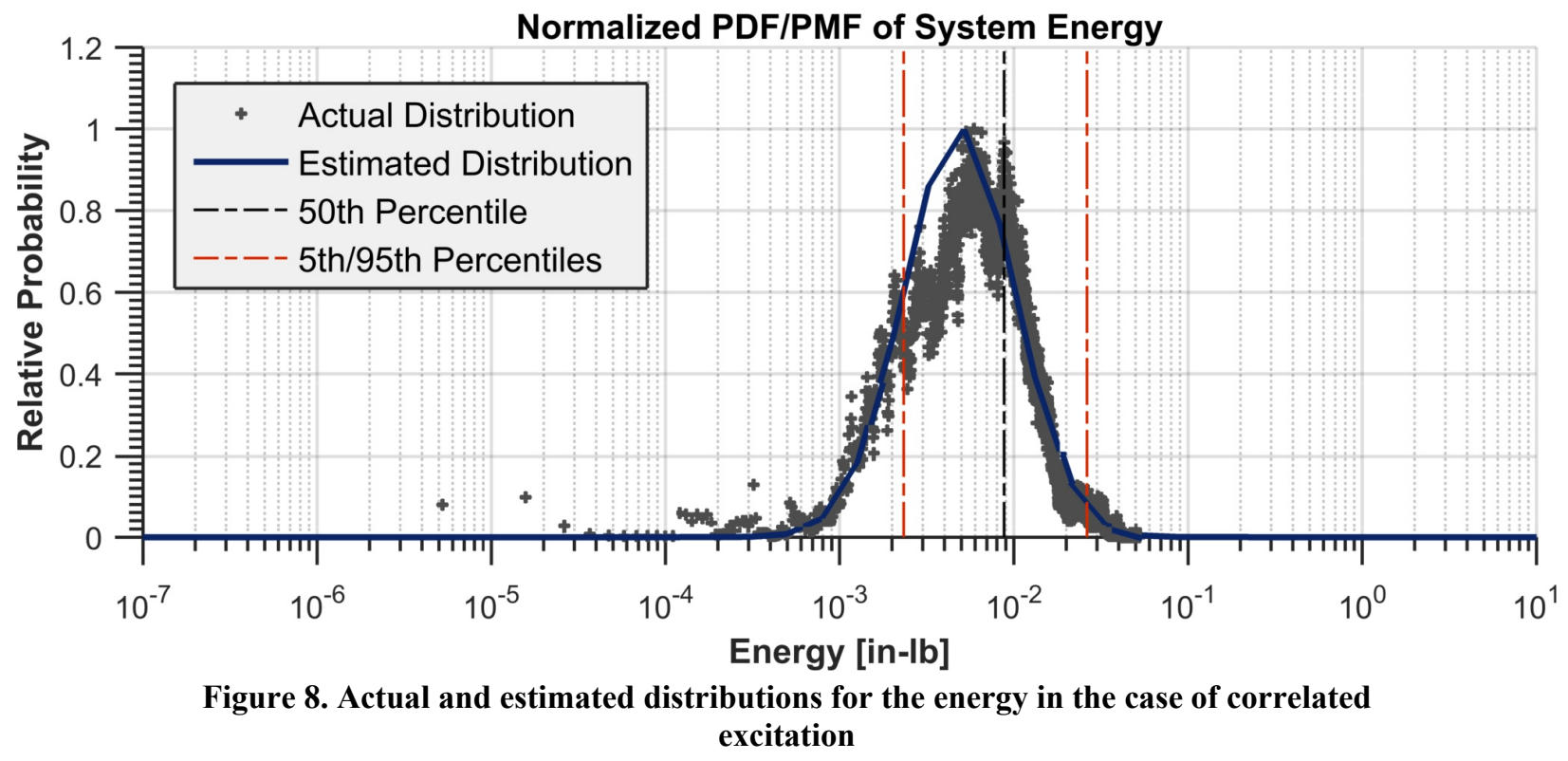

Figure 9 shows the beam's response to this excitation, as simulated using a full order model and a variety of reduced order models: A ROM with modes $(1,2,3,4)$, as well as the first four single-mode models, is plotted along with the full-order solution. Since the forcing is no longer symmetric, it is the power spectral density of the node at $\mathrm{x}$ $=\mathrm{L} / 3$ that is shown, allowing examination of asymmetric modes.

As in the previous example, the distribution of energy and the NNM curves were used to predict the width of each resonance peak, and the predicted widths (vertical lines) are compared with the actual spectrum in Fig. 9a. Two interesting points are immediately of note. The first NNM's 50th percentile frequency is almost identical to that of its 5th percentile frequency, leading to an asymmetric bracket for the mode. More significantly, while the bounds give a reasonable estimate of the width of the peaks in the spectrum for modes 1-3, the fourth NNM was predicted to behave completely linear, and yet the response near the $4^{\text {th }}$ mode shows significant smearing. While the erroneous prediction for this mode could, perhaps, be explained as in the previous sections, it is clear that superposition does not hold for this mode.

Several one-mode models were also used to predict the corresponding part of the response spectrum. In this case modes one and two have about equal strength, which contrasts the previous case where one mode was clearly dominant. As a result, in this case modes one and two could each be used alone to predict the corresponding part of the spectrum, suggesting that these modes are not strongly coupled by the nonlinearity. As in the previous case, the ROMs created with a higher frequency mode (modes 3 and 4 in this case) are far too linear and greatly in error compared to the truth solution. 

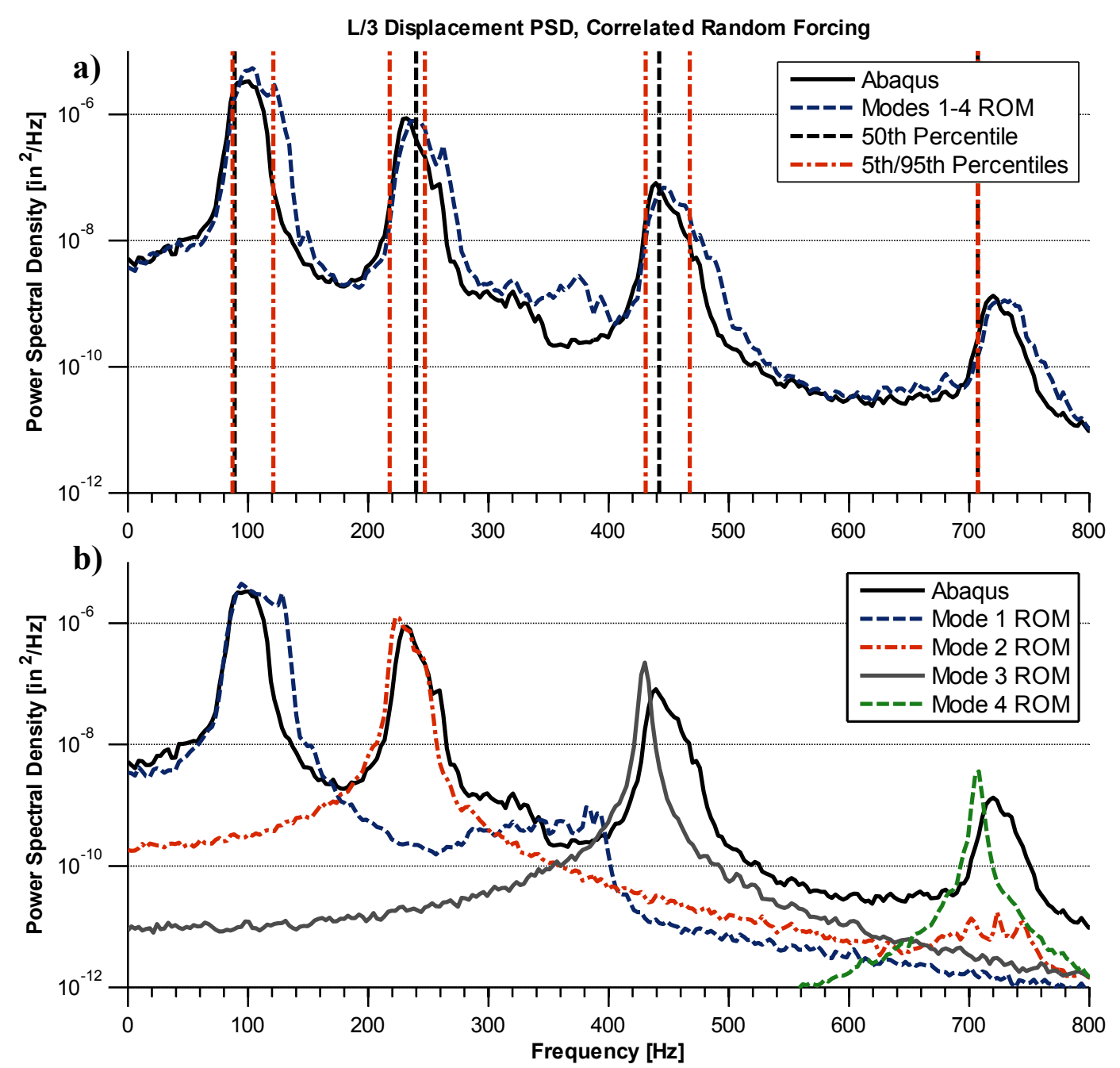

Figure 9. Power spectral density comparisons of full and reduced-order models using a random forcing correlated with position. (a) Four-mode ROM. (b) Single-mode ROMs.

\section{Conclusions}

This work has explored the connection between the random response of a structure and its undamped nonlinear normal modes. Simulations illustrated that the response to random input of each mode of the nonlinear structure is smeared over a range of frequencies when the excitation force is large, as expected due to the stiffening effect of the geometric nonlinearity. Furthermore, for the examples studied here, the degree of spreading in the power spectrum could be predicted to some extent using the frequency-energy plot of the structure's NNMs. Three methods were explored to quantify this, and each provided a unique perspective regarding the nonlinear response: 1.) the concept of equipartition was postulated to hold for these nonlinear structures and used to predict the frequency smearing based on the distribution of energy in the structure, 2.) Reduced order models were created that reproduced each mode individually (neglecting modal coupling), 3.) A linearized model was used in which the structure was prestressed into the instantaneous deformation of the first (dominant) mode at each instant in time. Interestingly, the second method greatly under-predicted the smearing in the higher modes, presumably because the ROM did not absorb enough energy to excite the structure into a nonlinear state. However, it did provide surprisingly accurate estimates of the strain in the structure due to a higher mode (see Fig. 7). Method three predicted the frequency distribution well, and taken together these findings suggest that the higher modes can be considered to behave linearly about the deformation state induced by the dominant mode.

One purpose of these comparisons was to explore the effect of modal coupling on the random response. In particular, the effect of internal resonances was studied by comparing the random response of ROMs that included 
or excluded coupling between certain modes, and hence included/excluded the associated internal resonance branches on their NNMs (i.e. see Figs 3, 6 and 7.). The random response was also computed at various excitation amplitudes to see whether the internal resonances would manifest themselves in the PSD as the response amplitude became large enough to encompass the internal resonances, i.e. see Fig. 7. The corresponding PSDs showed no interesting features and hence were not included here. Indeed, for the structure studied here it seems that internal resonances do not have a direct bearing on the response but are only important in that they are indicative of what modal couplings each ROM can describe. Perhaps this seems reasonable if we recognize that, as elaborated in Appendix A, the internal resonances do not necessarily indicate a dramatic change in the underlying vector field but simply identify special paths in the state space about which the system may depart from the fundamental manifold while still exhibiting a periodic orbit.

In any event this investigation clearly shows that a structure's forced, random response is intimately connected to its nonlinear normal modes. Further, if a reduced order model is capable of accurately predicting the frequencyenergy backbone of the nonlinear modes in the frequency-energy range of interest, then the results presented here suggest that it will also accurately predict the random response of a nonlinear structure in that same range of frequency and energy. While this claim cannot be proven generally, it seems reasonable based on the theory reviewed here. One may question whether it is advantageous to validate a reduced order model using NNMs rather than its PSDs. On the one hand the PSD is a physical quantity that is meaningful for design, and it has been used previously to validate models (see, e.g. [1]). However, the PSD depends on response amplitude so the comparison must be performed with various loading scenarios and while monitoring the response at many points. In contrast, the NNMs are deterministic and so they may be less expensive to compute over a range of energy than a PSD. The NNMs are also a global property of the structure, so if the relevant NNMs are accurate then it seems reasonable that the responses anywhere on the structure would also be accurate. The NNMs are also independent of the shape of the random input and one NNM describes the response at a large range of excitation levels.

It was also interesting to note that the response of the geometrically nonlinear beam studied here was dominated by one or two modes and hence one could predict the overall response or strain quite accurately considering only one mode. This finding could be used to develop a highly efficient design tool. For example, using an analytical solution for the SDOF differential equation one could very rapidly explore the effect of design changes on the RMS response. The computational savings could be very significant; indeed, about 12 hours were required to compute the PSD of the full order finite element model for the simple beam that was studied here, which had only 117 DOF! The random response of a one-mode ROM can be computed in a minute or so using numerical integration, and the analytical solutions in [23] can be evaluated in a tiny fraction of a second. Of course, to date we have only considered only a few structures that have low modal density. Certainly the findings in [24] suggest that multi-mode effects can become very important for modally dense structures. Further research should be performed to better understand when this type of simplification can be relied on and when multi-mode models should be considered.

\section{Acknowledgments}

This work was supported by the Air Force Office of Scientific Research, Award \# FA9550-11-1-0035, under the Multi-Scale Structural Mechanics and Prognosis program managed by Dr. David Stargel. Also, the authors would like to thank Joseph Hollkamp and other collaborators in the Structural Sciences Center at the Air Force Research Laboratory for many helpful suggestions and discussions. Additional support came from the National Science Foundation under Grant Nos. CMMI-0969224 and DGE-1256259 with the associated REU supplements. Any opinions, findings, and conclusions or recommendations expressed in this material are those of the authors(s) and do not necessarily reflect the views of the National Science Foundation. 


\section{Appendix}

\section{A. Relationships between NNMs, Invariant Manifolds and Vector Field}

\section{Theory}

Shaw and Pierre presented a definition in which damped nonlinear modes are defined as invariant manifolds in the state space, or solutions to the damped, nonlinear equation of motion. In this description, any response that starts on one of the NNM manifolds will stay on that manifold for all time (i.e. it is described by a single second order differential equation). While this work will focus on the undamped NNM definition used by Vakakis and Kerschen $[5,6]$, it is important to note that when the damping is light, as is typical for structural systems, the undamped NNMs can be stitched together to obtain a very good estimate of the damped NNM manifold.

This is illustrated in Fig. 10 below, where the frequency-energy curve for the undamped Duffing equation,

$$
\ddot{x}+x+0.5 x^{3}=0
$$

is shown. In this case the manifold encompasses the whole $x, d x / d t$ plane. (In general the NNM defines a plane in a higher dimensional space.) A random forcing function can be thought of as introducing distortions to the vector field and those distortions are random functions of time. If the forcing is small then one can see why the response tends to follow an NNM.
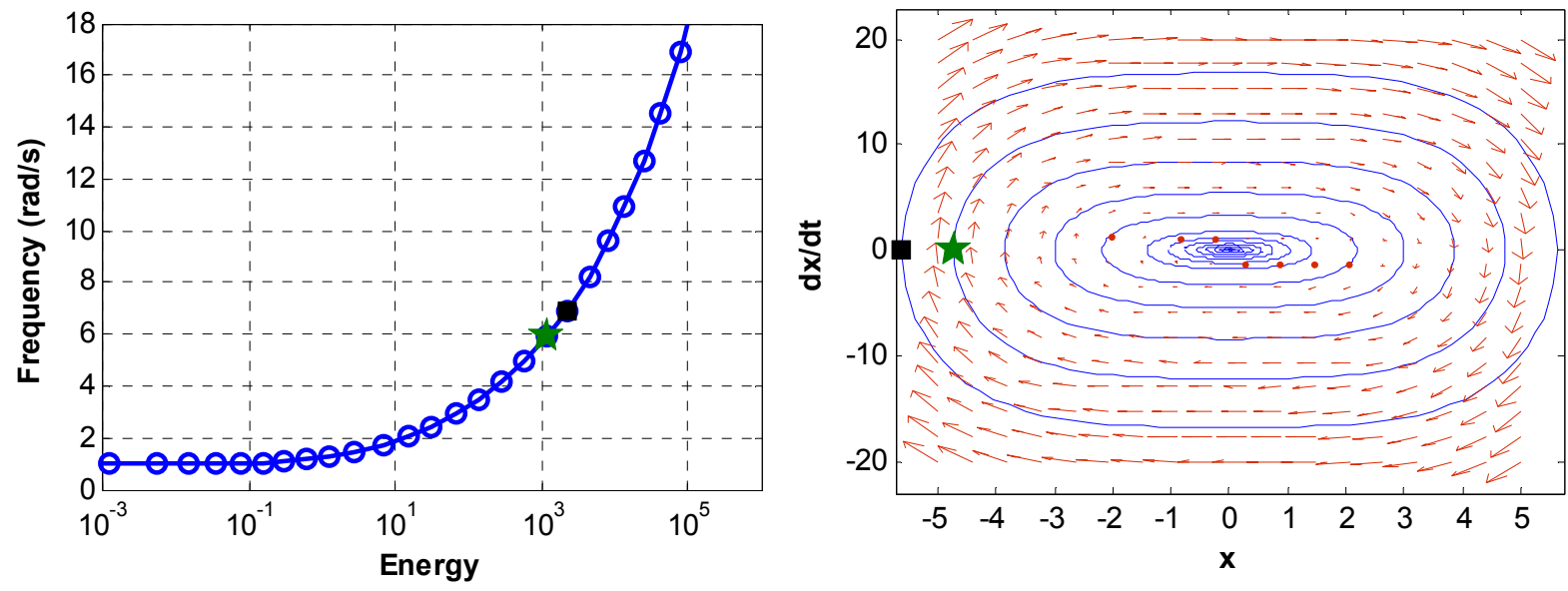

Figure 10. (a) Frequency-energy plot of the Duffing system described by Eq. (A1). (b) Graphical representation of the vector field (red) with several solutions overlaid (blue). These solutions form an NNM manifold, which for this SDOF system encompasses the whole $x, d x / d t$ plane.

\section{Application to 2-DOF Reduced Order Model}

It is informative to see how these concepts extend to higher order systems. Figure 11 shows portions of the manifold for NNM-1, computed from the mode $(1,3)$ reduced order model in Section IV. The coordinate axes are the modal displacements of linear modes 1 and 3 and the modal velocity of mode 1. At low energy, Fig. 11(a), shows that all of the orbits remain in the $q_{1}, d q_{1} / d t$ plane. At higher energies the linear modal coordinates couple and the surface deforms into the $q_{3}$ direction. Figure 11(b) shows the same manifold over a larger range of displacements. The internal resonance is manifest as an ellipsoid, where the responses extending into the $q_{3}$ direction lie on the surface of an ellipsoid. As in the previous example, if the vector field were shown it would again always be tangent to the NNM orbits, and hence this example illustrates that there are other paths away from the fundamental NNM manifold for which the response is always tangent to the vector field. 

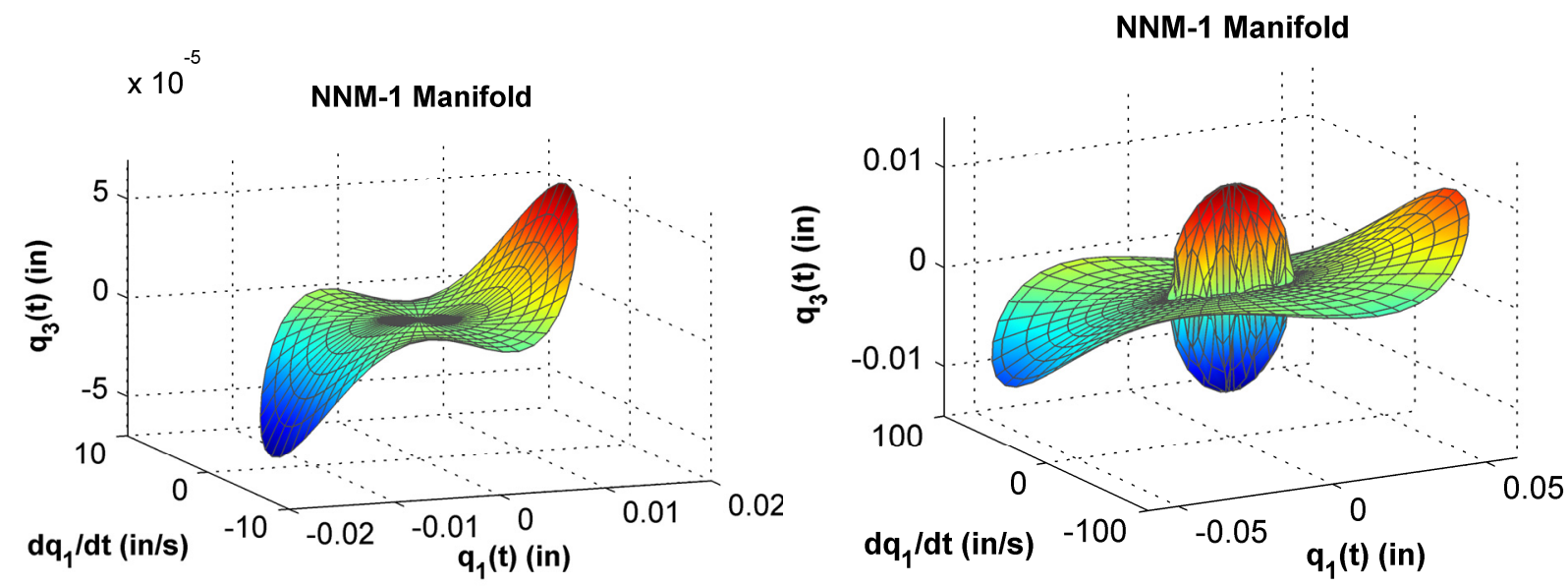

Figure 11. (a) 3D Poincare section of the NNM manifold for the mode [1,3] ROM in Fig. 6 corresponding to $d q_{3} / d t=0$. (b) Same, over a wider range of response amplitude. The internal resonance is visible where the manifold forms an ellipsoid extending into the $q_{3}$ direction.

A simpler way to visualize the manifolds for this system is in a 2D Poincare section, as shown in Fig. 12 . It is clear that there are regions in the state space where a small perturbation from the random force field could cause the system to follow a path where linear modes 1 and 3 are both active. Hence, it is interesting to note that the internal resonances do not feature prominently in the random responses shown in Figs 2 and 3.

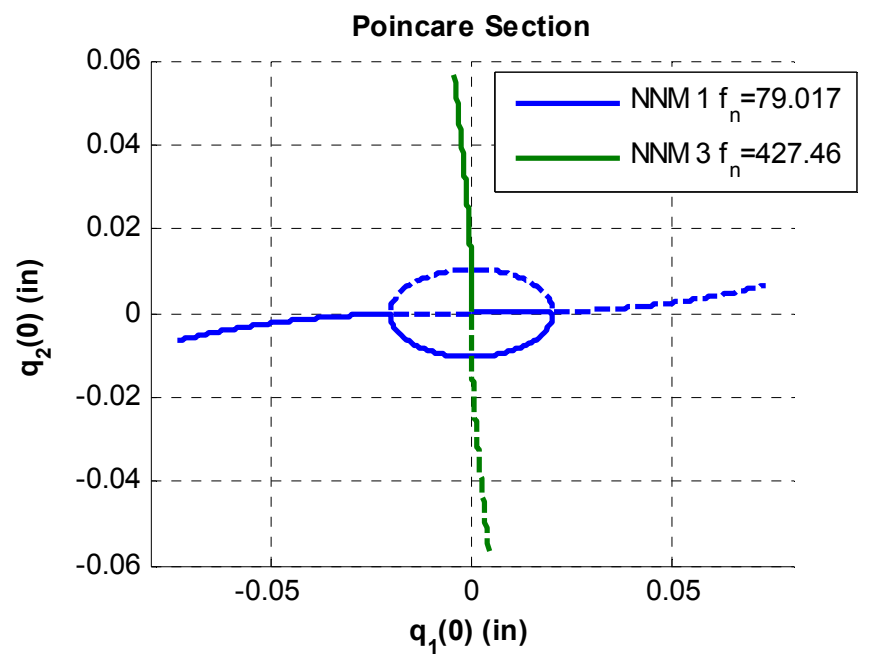

Figure 12. 2D Poincare section of the NNM manifold for the mode [1,3] ROM in Fig. 6 corresponding to $d q_{1} / d t=d q_{3} / d t=0$. The portions shown with dashed lines correspond to cases where the initial conditions are the negative of those shown with solid lines.

\section{B. Frequency Smearing Explained by Linearizing about a Dominant Mode}

An examination of mode 1's effect on the frequency shift and spread of higher modes was conducted. Qualitatively, this effect is a result of the stiffening behavior generated by a deflection in mode 1; it was postulated that, since mode 1 was dominant in the responses studied, the nonlinearity seen in the frequency response of higher modes was dependent on this mode. To accomplish this goal, the undamped free response was first considered. An alternative formulation of a reduced order model containing $m$ modes is given by Nash [16],

$$
\mathbf{I}_{m} \ddot{\mathbf{q}}+\left[\boldsymbol{\Lambda}_{m}+\frac{1}{2} \mathbf{N}_{1}(\mathbf{q})+\frac{1}{3} \mathbf{N}_{2}(\mathbf{q})\right] \mathbf{q}=\mathbf{0}
$$


Where $\mathbf{I}_{m}$ is the identity matrix of order $m$ and $\boldsymbol{\Lambda}_{m}$ contains the squared natural frequencies of each included mode along its diagonal. $\mathbf{N}_{1}(\mathbf{q})$ and $\mathbf{N}_{2}(\mathbf{q})$ are $m \times m$ nonlinear stiffness matrices which correspond to quadratic and cubic nonlinearities, respectively. Defining these matrices is a two-step process. First, a vector of cubic terms must be constructed. (The quadratic terms correspond to softening-type nonlinearities and are uniformly zero for perfectly flat structures such as this. When required - in curved structures, for instance - they can treated in a similar manner).

$$
\boldsymbol{\alpha}=\left[\begin{array}{llll}
\alpha_{1} & \alpha_{2} & \ldots & \alpha_{m}
\end{array}\right]^{T} \quad \text { where } \quad \alpha_{i}=\sum_{i=1}^{m} \sum_{j=1}^{m} \sum_{k=1}^{m} A_{i}(i, j, k) q_{i} q_{j} q_{k}
$$

The nonlinear stiffness matrix is then the Jacobian matrix of $\boldsymbol{\alpha}$ with respect to $\mathbf{q}$.

$$
\mathbf{N}_{2}(\mathbf{q})=\frac{\partial \boldsymbol{\alpha}}{\partial \mathbf{q}}
$$

To linearize the system about a particular deflected state of the first mode, the linear stiffness was found about a particular deflection state of mode 1, i.e. by setting $\mathbf{q}_{0}=\left[\begin{array}{lllll}q_{1} & 0 & \ldots & 0\end{array}\right]^{T}$.

$$
\mathbf{K}_{e q}=\left(\frac{\partial}{\partial \mathbf{q}}\left[\boldsymbol{\Lambda}_{m}+\frac{1}{2} \mathbf{N}_{1}(\mathbf{q})+\frac{1}{3} \mathbf{N}_{2}(\mathbf{q})\right] \mathbf{q}\right)_{\mathbf{q}=\mathbf{q}_{0}}
$$

Equation (A2) can now be written as a linear system, from which the modified natural frequencies can be extracted by solving the eigenvalue problem for the equation of motion given below. This then gives each of the natural frequencies of the structure for a given level of deflection in the dominant mode.

$$
\mathbf{I}_{m} \ddot{\mathbf{q}}+\mathbf{K}_{e q} \mathbf{q}=\mathbf{0}
$$

For both loading cases tested in this work, each mode's mean frequency was an excellent approximation to the frequency of the peak in the nonlinear response (i.e. in Fig. 6), and the standard deviation of each mode served as a useful measure of the width of each response peak. Specifically, the mean and standard deviation of mode 1's response amplitude was found based on the 1-mode ROM in Fig. 9. These were then used in Eq. (A5-A6) to compute the pre-stressed natural frequencies of modes 1,2,3, and 4 at the mean and at the mean plus or minus one standard deviation. The corresponding frequencies are shown by dashed lines in Fig.13. This shows that the extent of frequency smearing in mode 3 and mode 4's peaks are well-predicted by this linearization method, while the 2nd mode's peak spreads a little more than this would predict. This indicates that the nonlinearity in modes 3 and 4 are may simply be caused by pre-stress due to mode 1 deflections, while mode 2's actual response may also depend on other factors such as modal coupling. Similar results were found (not shown here) for the uniform loading case in Fig. 6. In that case the smearing for modes 3,5 and 7 was well predicted by the linearized model. These results suggest that the smearing in the higher mode frequencies can be explained simply by a changing state of pre-stress rather than an exchange of energy from low frequency modes to higher frequencies. 


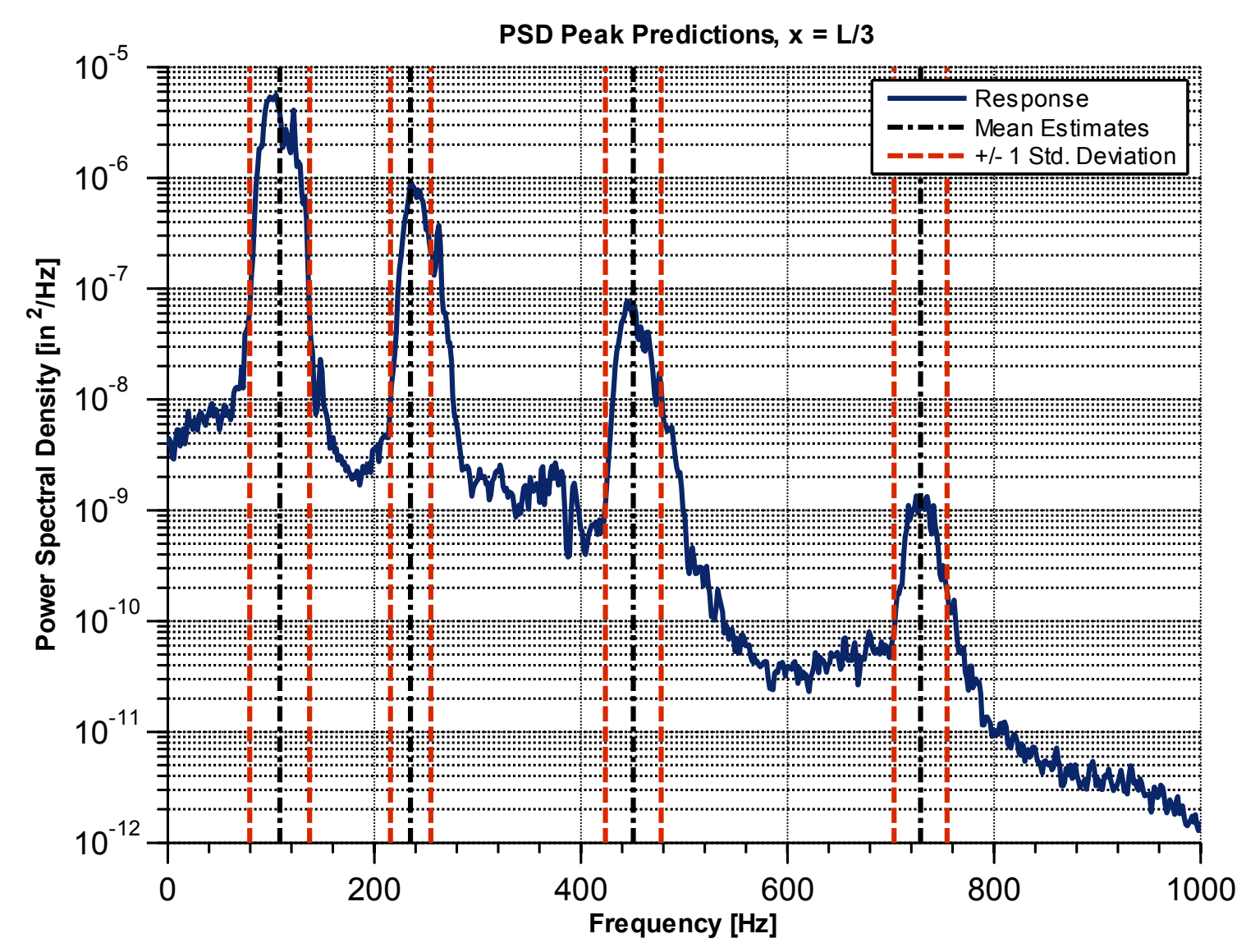

Figure 13. Peak prediction brackets obtained via linearizing the stiffness matrix about mode 1's instantaneous deflection

\section{References}

[1] R. W. Gordon and J. J. Hollkamp, "Reduced-order Models for Acoustic Response Prediction," Air Force Research Laboratory, AFRL-RB-WP-TR-2011-3040, Dayton, OH2011.

[2] J. J. Hollkamp and R. W. Gordon, "Reduced-order models for nonlinear response prediction: Implicit condensation and expansion," Journal of Sound and Vibration, vol. 318, pp. 1139-1153, 2008.

[3] T. Beberniss, T. Eason, B. Gordon, M. Haney, J. Hollkamp, and S. M. Spottswood, "Response Prediction for Structures in Extreme Environments," Structural Sciences Center, AFRL/VASM, Dayton, Oh15 July 20072007.

[4] M. P. Mignolet, A. Przekop, S. A. Rizzi, and S. M. Spottswood, "A review of indirect/non-intrusive reduced order modeling of nonlinear geometric structures," Journal of Sound and Vibration, vol. 332, pp. 2437-2460, 2013.

[5] G. Kerschen, M. Peeters, J. C. Golinval, and A. F. Vakakis, "Nonlinear normal modes. Part I. A useful framework for the structural dynamicist," Mechanical Systems and Signal Processing, vol. 23, pp. 170-94, 2009.

[6] A. F. Vakakis, "Non-linear normal modes (NNMs) and their applications in vibration theory: an overview," Mechanical Systems and Signal Processing, vol. 11, pp. 3-22, 1997.

[7] R. M. Rosenberg, "Normal modes of nonlinear dual-mode systems," Journal of Applied Mechanics, vol. 27, pp. 263-268, 1960.

[8] T. L. Hill, A. Cammarano, S. A. Neild, and D. J. Wagg, "Interpreting the forced responses of a two-degreeof-freedom nonlinear oscillator using backbone curves," Journal of Sound and Vibration, vol. 349, pp. 276$288,2015$. 
[9] H. A. Ardeh and M. S. Allen, "Instantaneous Center Manifolds and Nonlinear Modes of Vibration," presented at the ASME 2012 International Design Engineering Technical Conferences, Chicago, IL, 2012.

[10] H. A. Ardeh and M. S. Allen, "Investigating Cases of Jump Phenomenon in a Nonlinear Oscillatory System," presented at the 31st International Modal Analysis Conference (IMAC XXXI), Garden Grove, CA, 2013.

[11] B. K. Øksendal, Stochastic Differential Equations: An Introduction with Applications. Berlin: Springer, 2003.

[12] D. A. W. Barton, S. G. Burrow, and L. R. Clare, "Energy Harvesting From Vibrations With a Nonlinear Oscillator," Journal of Vibration and Acoustics, vol. 132, p. 021009 (7 pp.), 2010.

[13] P. Kumar and S. Narayanan, "Modified path integral solution of Fokker-Planck equation: response and bifurcation of nonlinear systems," Journal of Computational and Nonlinear Dynamics, vol. 5, p. 011004 (12 pp.), 2010.

[14] E. J. Cross and K. Worden, "Approximation of the Duffing oscillator frequency response function using the FPK equation," Journal of Physics: Conference Series, vol. 181, p. 012085 (9 pp.), 2009.

[15] M. Peeters, G. Kerschen, and J. C. Golinval, "Dynamic testing of nonlinear vibrating structures using nonlinear normal modes," Journal of Sound and Vibration, vol. 330, pp. 486-509, 2011.

[16] M. Peeters, G. Kerschen, and J. C. Golinval, "Modal testing of nonlinear vibrating structures based on nonlinear normal modes: Experimental demonstration," Mechanical Systems and Signal Processing, vol. 25, pp. 1227-1247, 2011.

[17] R. K. Pathria, Statistical Mechanics: Pergamon Press, 1972.

[18] J. E. Sader, I. Larson, P. Mulvaney, and L. R. White, "Method for the calibration of atomic force microscope cantilevers," Review of Scientific Instruments, vol. 66, pp. 3789-3798, 1995.

[19] R. J. Kuether and M. S. Allen, "A Numerical Approach to Directly Compute Nonlinear Normal Modes of Geometrically Nonlinear Finite Element Models," Mechanical Systems and Signal Processing, vol. 46, pp. $1-15,2014$.

[20] M. S. Allen, R. J. Kuether, B. Deaner, and M. W. Sracic, "A Numerical Continuation Method to Compute Nonlinear Normal Modes Using Modal Reduction," presented at the 53rd AIAA Structures, Structural Dynamics, and Materials Conference, Honolulu, Hawaii, 2012.

[21] M. Peeters, R. Viguie, G. Serandour, G. Kerschen, and J. C. Golinval, "Nonlinear normal modes, part II: toward a practical computation using numerical continuation techniques," Mechanical Systems and Signal Processing, vol. 23, pp. 195-216, 2009.

[22] R. J. Kuether, B. Deaner, M. S. Allen, and J. J. Hollkamp, "Evaluation of Geometrically Nonlinear Reduced Order Models with Nonlinear Normal Modes " AIAA Journal, vol. 53, pp. 3273-3285, 2015.

[23] L. D. Lutes and S. Sarkani, Random vibrations: analysis of structural and mechanical systems. New York: Elsevier Butterworth-Heinemann, 2004.

[24] G. M. Spelman and R. S. Langley, "Statistical energy analysis of nonlinear vibrating systems," Philosophical Transactions of the Royal Society A: Mathematical, Physical and Engineering Sciences, vol. 373, 2015. 